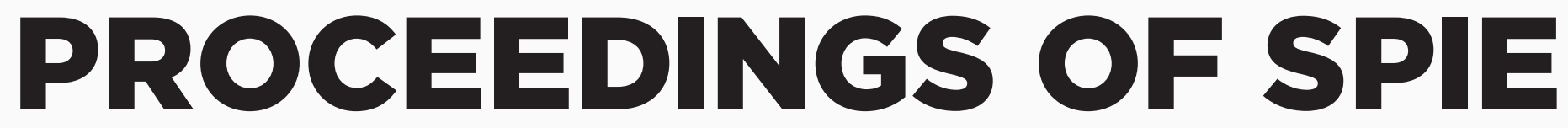

\title{
Three-dimensional transfer functions
}

\section{Colin Sheppard}

Colin J. R. Sheppard, "Three-dimensional transfer functions," Proc. SPIE 3831, Sixth International Conference on Education and Training in Optics and Photonics, (16 June 2000); doi: 10.1117/12.388717

Event: Education and Training in Optics and Photonics (ETOP'99), 1999, Cancun, Mexico 


\title{
Three-dimensional transfer functions
}

\author{
Colin J. R. Sheppard*a,b \\ aPhysical Optics Department, School of Physics, University of Sydney, \\ NSW 2006, Australia \\ bAustralian Key Centre for Microscopy and Microanalysis,University of Sydney, \\ NSW 2006, Australia
}

\begin{abstract}
The optical transfer function (OTF) is widely used to investigate the focusing and imaging properties of an optical system, including image modeling, comparison of relative imaging performance, and image reconstruction. An optical system can form a threedimensional (3-D) image of a 3-D object, and the 3-D OTF is a useful approach to investigating the behaviour. The 3-D transfer function is particularly useful for study of image formation in confocal and interference microscopes. As the OTF represents the power spectral density it is also useful for studying beam propagation and scattering. The connection with the ambiguity function is also described.
\end{abstract}

Keywords: Diffraction, focusing, imaging, microscopy, transfer functions, beam propagation, scattering, ambiguity function.

\section{INTRODUCTION}

An optical system can be used to form a 3-D image. According to Helmholtz and Maxwell it is impossible to form a perfect image of a 3-D object. But if the object is scanned in the axial direction a perfect image can be formed in a computer.

The concept of the OTF is widely used in many branches of optics. It can be used to quantify the imaging performance of an optical system, or to represent the power spectral density in beam propagation. Many of these principles can be directly generalised to the 3D case. Consider an optical system with pupil function $P_{0}$. The amplitude point spread function is given by the Fourier transform of the pupil function. In a coherent imaging system, imaging can be characterised by a coherent transfer function, which is, apart from a scaling, equal to the pupil function. We can thus regard the pupil function $P_{0}(m)$ as a function of normalised spatial frequencies $m, n$ so that

$$
P_{0}(m)=0, \quad|m|>1 .
$$

If the system is defocused, the defocused pupil function for a paraxial system is

$$
P(m, n, u)=P_{0}(m, n) \exp \left(\frac{1}{2} i u\left(m^{2}+n^{2}\right)\right)
$$


in which $u$ is a normalised axial displacement

$$
u=4 k z \sin ^{2} \frac{\alpha}{2}
$$

where $\alpha$ is the angular aperture of the lens and $k=2 \pi / \lambda$. Then the defocused OTF is given by the autocorrelation of the defocused pupil function

$$
\begin{aligned}
& C(m, n, u)=\frac{\int P\left(m^{\prime}+m / 2, n^{\prime}+n / 2\right) P^{*}\left(m^{\prime}-m / 2, n^{\prime}-n / 2\right) \mathrm{d} m^{\prime} \mathrm{d} n^{\prime}}{\int\left|P\left(m^{\prime}, n^{\prime}\right)\right|^{2} \mathrm{~d} m^{\prime} \mathrm{d} n^{\prime}} \\
& =\frac{1}{E} \int P_{0}\left(m^{\prime}+m / 2, n^{\prime}+n / 2\right) P_{0}^{*}\left(m^{\prime}-m / 2, n^{\prime}-n / 2\right) \exp \left(i u\left(m m^{\prime}+n n^{\prime}\right)\right) \mathrm{d} m^{\prime} \mathrm{d} n^{\prime}
\end{aligned}
$$

where $E$ is the energy in the beam. All integrals are taken to be evaluated from minus to plus infinity.

\section{PARAXIAL 3-D TRANSFER FUNCTIONS}

Consider now the 3-D pupil function, given by the Fourier transform of the defocused pupil function,

$$
\Pi(m, n, s)=P_{0}(m, n) \int \exp \left(\frac{1}{2} i u\left(m^{2}+n^{2}\right)\right) \exp (-i u s) \mathrm{d} u
$$

Then

$$
\Pi(m, n, s)=P_{0}(m, n) \delta\left(s-\left(m^{2}+n^{2}\right) / 2\right)
$$

so that it lies on a paraboloid, and also

$$
P_{0}(m, n)=\int \Pi(m, n, s) \mathrm{d} s
$$

The concept of the 3-D pupil was introduced by McCutchen ${ }^{1}$ The amplitude point spread function is the 3-D Fourier transform of the 3-D pupil function. Coherent 3-D imaging can be described in terms of a 3-D coherent transfer function, given by a scaled 3-D pupil function. This concept was first introduced by Wolf. ${ }^{2}$

In the same way, the 3-D OTF for 3-D imaging in an incoherent system is given by the 3-D Fourier transform of the defocused $\mathrm{OTF}^{3,4}$ 


$$
C_{2}(m, s)=\int C(m, u) \exp (-i u s) \mathrm{d} u
$$

The 3-D OTF is given by the autocorrelation of the 3-D pupil function, 5,6 or by a line integral on a straight path across the region of overlap of two displaced pupils, as originally pointed out by Frieden. ${ }^{4}$ For a circular pupil it exhibits a singularity at the origin, apart from which it is zero along the $s$ axis, so that lamellar structures cannot be imaged.

Coherent imaging of a thick object can be studied using the scattering function $S\left(m_{2}, n_{2} ; m_{1}, n_{1}\right)$, which describes scattering from directions corresponding to $m_{1}, n_{1}$ to $m_{2}, n_{2} \cdot{ }^{7}$ In some special cases, the scattering function reduces to a function of three variables $T(m, n, s)$. These cases include those when the Born or Kirchhoff approximations are valid, for spherical symmetry, or for a thin planar object. Imaging of a general 3-D object is then considered by expanding the object into 3-D gratings and using the 3-D coherent transfer function.

Confocal or interference microscopes behave completely differently from conventional imaging systems in 3-D imaging. The coherent transfer function is no longer confined to a surface, but is non-zero over a region of 3-D spatial frequencies. ${ }^{8-10}$ Similarly, the 3-D OTF does not exhibit a singularity, and is non-zero along the $s$ axis. ${ }^{11,12}$

The effects of an annular pupil on the 3-D transfer function have also been investigated, ${ }^{13-15}$ as have the effects of aberrations ${ }^{16-18}$

The paraxial treatment can also be extended to the high-aperture case, when the parabolic surface of the paraxial case becomes the surface of the Ewald sphere. $5,6,8,9,19$ The full vectorial case has also been considered. ${ }^{20}$

\section{CONNECTION WITH THE AMBIGUITY FUNCTION}

For a 2-D system, i.e. with cylindrical lenses, the defocused OTF of a lens can be expressed directly in terms of the ambiguity function. ${ }^{21}$ The ambiguity function is defined as ${ }^{22}$

$$
A(m, x)=\frac{1}{E} \int P_{0}\left(m^{\prime}+m / 2\right) P_{0}^{*}\left(m^{\prime}-m / 2\right) \exp \left(\mathrm{i} 2 \pi m^{\prime} x\right) \mathrm{d} m^{\prime}
$$

Comparing Eqs. 4 and 9, we thus have a relationship between the defocused OTF and the ambiguity function, ${ }^{21}$ with

$$
u m=2 \pi x
$$

Papoulis $^{22}$ also considers the spectral correlation function 


$$
\gamma\left(m, m^{\prime}\right)=\frac{1}{E} P_{0}\left(m^{\prime}+m / 2\right) P^{*}\left(m^{\prime}-m / 2\right)
$$

so that we have

$$
C(m, u)=A\left(m, \frac{u m}{2 \pi}\right)=\int \gamma\left(m, m^{\prime}\right) \exp \left(i u m m^{\prime}\right) \mathrm{d} m^{\prime}
$$

The 2-D OTF is given in terms of the ambiguity function, using Eq. 12

$$
C_{2}(m, s)=\frac{2 \pi}{m} \int A(m, x) \exp \left(-\frac{i 2 \pi x s}{m}\right) \mathrm{d} x
$$

which can be inverted to give

$$
A(m, x)=\frac{1}{2 \pi} \int C_{2}(m, s) \exp \left(\frac{i 2 \pi x s}{m}\right) \mathrm{d} s
$$

Comparing with Eq. 12, we then have

so that

$$
s / m=m^{\prime}
$$

$$
\gamma\left(m, m^{\prime}\right)=\frac{m}{2 \pi} C_{2}\left(m, m m^{\prime}\right)
$$

or

$$
C_{2}(m, s)=\frac{2 \pi}{m} \gamma\left(m, \frac{s}{m}\right)
$$

This treatment can be extended to the case of 2-D pupils. Then the spectral correlation function is

$$
\gamma\left(m, m^{\prime} ; n, n^{\prime}\right)=\frac{1}{E} P_{0}\left(m^{\prime}+m / 2, n^{\prime}+n / 2\right) P_{0}^{*}\left(m^{\prime}-m / 2, n^{\prime}-n / 2\right)
$$

The ambiguity function is

$$
A(m, x ; n, y)=\iint \gamma\left(m, m^{\prime} ; n, n^{\prime}\right) \exp \left(i 2 \pi\left(m^{\prime} x+n^{\prime} y\right)\right) \mathrm{d} m^{\prime} \mathrm{d} n^{\prime}
$$

Comparing Eqs. 25, and 26 we have 


$$
C(m, n, u)=A\left(m, \frac{u m}{2 \pi} ; n, \frac{u n}{2 \pi}\right)
$$

Thus knowledge of the defocused OTF determines only a 3-D section through the 4-D ambiguity function.

The 3-D OTF is given by the Fourier transform of $C(m, n, u)$ with respect to $u$, giving

$$
C_{3}(m, n, s)=2 \pi \iint \gamma\left(m, m^{\prime} ; n, n^{\prime}\right) \delta\left(\left(s-\left(m m^{\prime}+n n^{\prime}\right)\right) \mathrm{d} m^{\prime} \mathrm{d} n^{\prime}\right.
$$

showing that the 3-D OTF is given by a line integral on a straight path across the region of overlap of two displaced pupils. ${ }^{4}$ The 3-D OTF can also be expressed in terms of the ambiguity function

$$
C_{3}(m, n, s)=\int A\left(m, \frac{u m}{2 \pi} ; n, \frac{u n}{2 \pi}\right) \exp (-i u s) \mathrm{d} u
$$

\section{ACKNOWLEDGMENTS}

The author acknowledges support from the Australian Research Council and the Science Foundation for Physics within the University of Sydney. Useful discussions with K. G. Larkin are acknowledged.

\section{REFERENCES}

1 C. W. McCutchen, "Generalized aperture and the three-dimensional diffraction image," J. Opt. Soc. Am. 54, 240-244, (1964).

2 E. Wolf, "Three-dimensional structure determination of semi-transparent objects from holographic data," Opt. Commun. 1, 153-156, (1969).

3 L. Mertz, Transformations in Optics, Wiley, New York, 1965.

4 B. R. Frieden, "Optical transfer of the three-dimensional object," J. Opt. Soc. Am. A 57, 56-66, (1967).

5 C. J. R. Sheppard, "General considerations of diffraction theory of 3-D imaging," European J. Cell Biology Suppl. 25, 29-32, (1989).

6 C. J. R. Sheppard, and C. J. Cogswell, "Three-dimensional image formation in confocal microscopy," J. Microsc. 159, 179-194, (1990).

7 C. J. R. Sheppard, and J. T. Sheridan, "Micrometrology of thick structures," Proc. SPIE 1139, 32-39, (1989).

8 C. J. R. Sheppard, "The spatial frequency cut-off in three-dimensional imaging," Optik 72, 131-133, (1986).

9 C. J. R. Sheppard, "The spatial frquency cut-off in three dimensional imaging II," Optik 74, 128-129, (1986).

10 C. J. R. Sheppard, M. Gu, and X. Q. Mao, "Three-dimensional coherent transfer function in a reflection-mode confocal scanning microscope," Opt. Commun. 81, 281-284, (1991). 
11 M. Gu, and C. J. R. Sheppard, "Confocal fluorescent microscopy with a finite-sized circular detector," J. Opt. Soc. Am. A 9, 151-153, (1992).

12 M. Gu, and C. J. R. Sheppard, "Effects of finite-sized detector on the OTF of confocal fluorescent microscopy," Optik 89,65-69, (1991).

13 C. J. R. Sheppard, and M. Gu, "Three-dimensional optical transfer function for an annular lens," Opt. Commun. 81, 276-280, (1991).

14 X. S. Gan, and C. J. R. Sheppard, "Imaging in a confocal microscope with one circular and one annular lens," Opt. Commun. 103, 254-264, (1993).

15 M. Gu, and C. J. R. Sheppard, "Three-dimensional coherent transfer functions in confocal imaging with two unequal annular lenses," J. Mod. Opt. 40, 1255-1272, (1993).

16 S. Wang, and B. R. Frieden, "Effects of third-order spherical aberration on the 3-D incoherent optical transfer function," Appl. Opt. 29, 2424-2432, (1990).

17 D. G. A. Jackson, M. Gu, and C. J. R. Sheppard, "3-D optical transfer function for circular and annular lenses with spherical aberration and defocus," J. Opt. Soc. Am. A 11, 1758-1767, (1994).

18 C. J. R. Sheppard, and M. Hole, "Three-dimensional optical transfer function for weak aberrations," J. Mod. Opt 42, 1921-1928, (1995).

19 C. J. R. Sheppard, M. Gu, Y. Kawata, and S. Kawata, "Three-Dimensional transfer functions for high aperture systems obeying the sine condition," J. Opt. Soc. Am. A 11, 593-598, (1994).

20 C. J. R. Sheppard, and K. G. Larkin, "Vectorial pupil functions and vectorial transfer functions," Optik 107, 79-87, (1997).

21 K.-H. Brenner, A. W. Lohmann, and J. Ojeda-Castanada, "The ambiguity function as a polar display of the OTF," Opt. Commun 44, 323-326, (1983).

22 A. Papoulis, "Ambiguity function in Fourier optics," J. Opt. Soc. Am. 64, 779788, (1974). 